\title{
Was Gelfand Right? The Many Loves of Lattice Theory
}
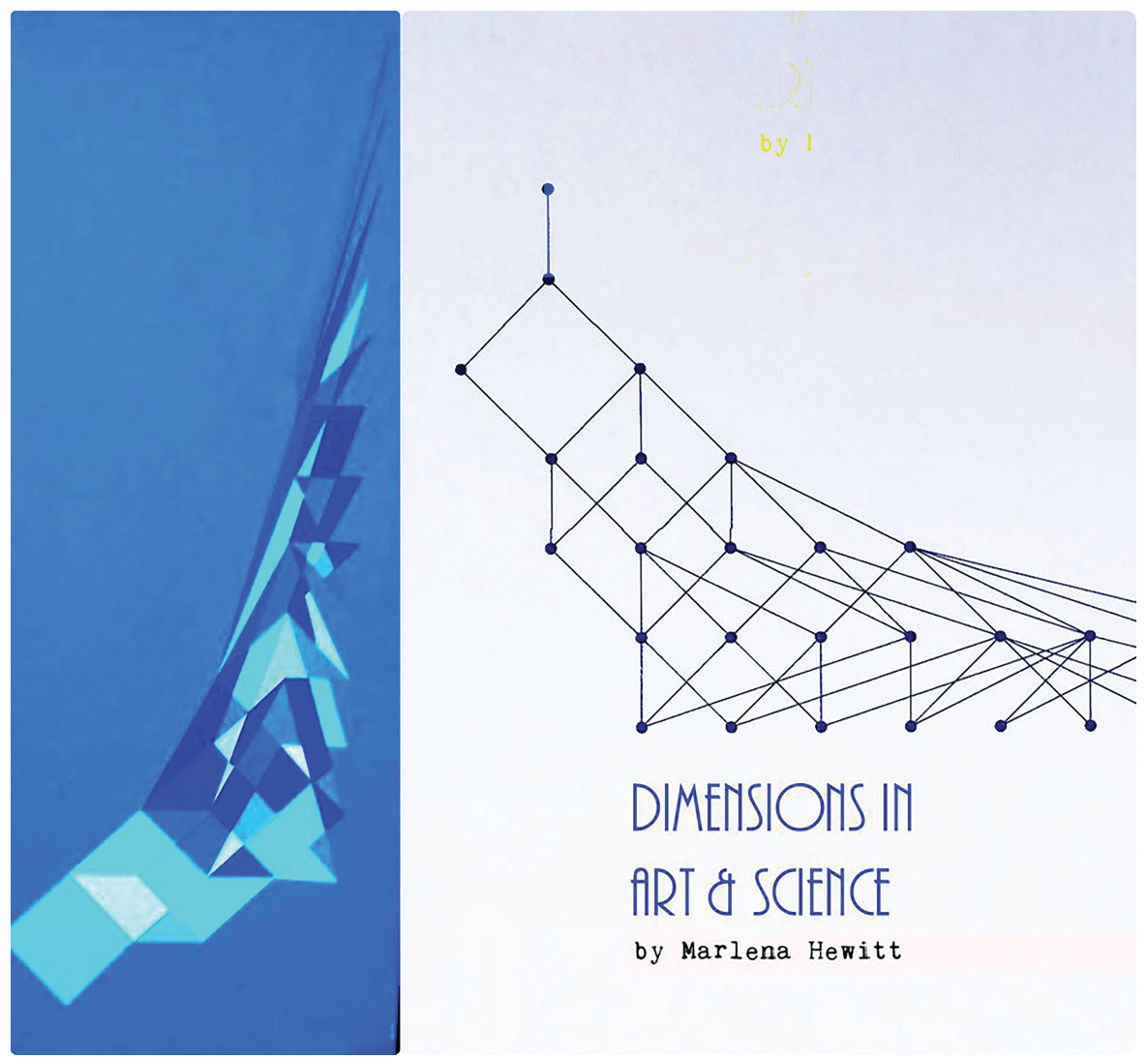

by Marlena Hewitt

\section{Jonathan David Farley}

Gian-Carlo Rota wrote in the Notices, "Never in the history of mathematics has a mathematical theory been the object of such vociferous vituperation as lattice theory." In this article, we outline some of the exciting open problems in lattice theory and the theory of ordered sets.

I was surprised when I walked into the office of the person I hoped would be my doctoral advisor. The year before, I had bought the book coauthored by that person, a light blue paperback I still have in my possession: Introduction to Lattices and Order, by B. A. Davey and H. A. Priestley.

Jonathan David Farley is an associate professor of mathematics at Morgan State University. His email address is 1attice. theory@gmai 1. com.

Communicated by Notices Associate Editor Emilie Purvine.

For permission to reprint this article, please contact:

reprint-permission@ams.org.

DOI: https://doi.org/10.1090/noti2415
Earlier that summer, there had been a conference in honor of Professor Garrett Birkhoff, who had been my undergraduate thesis advisor. At the conference, Professor Birkhoff told Dr. Priestley to expect me in the fall at the University of Oxford, where Dr. Priestley taught.

Dr. Priestley had sent me a handwritten note telling me we should meet at a particular office at the Mathematical Institute. The door was open and I walked in and that was the first time I realized that no one had actually used the pronoun "he" or "him," because sitting in a chair wearing a dress was the woman who would become my doctoral advisor, H. A. Priestley.

I had been confounded by the British practice of using initials, like J. R. R. Tolkien, C. S. Lewis, and H. R. Highness!

Like me, some mathematicians may have false preconceived notions, even prejudices, about lattice theorists, 
lattice theory, or the theory of ordered sets. I hope that what you find in these pages will instead surprise-and delight-and excite-you. Maybe you'll even find something you'll love.

\section{The Fixed-Point Property}

In the first conversation Dr. Priestley and I had, she described this problem:

Is the fixed-point property for posets preserved by products?

A partially ordered set-a.k.a. poset-is a pair $\left(P, \leq_{P}\right)$, where $P$ is a set and $\leq_{P}$ is a relation that is reflexive ( $p \leq p$ for all $p \in P$ ), transitive ( $p \leq q$ and $q \leq r$ imply $p \leq r$ for all $p, q, r \in P$ ), and antisymmetric ( $p \leq q$ and $q \leq p$ imply $p=q$ ). "Let $P$ be a poset" means we are considering $\left(P, \leq_{P}\right)$ for some set $P$ and relation $\leq_{P}$ on $P$ with those three properties.

Let $P$ and $Q$ be posets. A function $f: P \rightarrow Q$ is orderpreserving if $p \leq_{P} p^{\prime}$ implies $f(p) \leq_{Q} f\left(p^{\prime}\right)$. It is an orderembedding if also the converse holds. We denote the set of order-preserving maps from $P$ to $Q$ by $Q^{P}$. A poset $P$ has the fixed-point property if for every $f \in P^{P}$ there is a $p \in P$ such that $f(p)=p$.

We can define a partial ordering on the direct product of posets $P \times Q$ as follows: $(p, q) \leq_{P \times Q}\left(p^{\prime}, q^{\prime}\right)$ if and only if $p \leq_{P} p^{\prime}$ and $q \leq_{Q} q^{\prime}$. For example, if $\mathbf{n}$ is the $n$-element totally ordered set, then $\mathbf{2} \times \mathbf{2} \times \mathbf{2}$ is order-isomorphic to the power set of a three-element set.

Question 1. If $P$ and $Q$ have the fixed-point property, does $P \times Q$ ?

This problem was in circulation before it appeared in the first volume of the journal Order in 1984.

Roddy came up with the spectacular answer "Yes!"for finite posets-in 1994. Roddy sent me a letter saying that the key idea had been in Roddy's mind for a year, but Roddy couldn't figure out how to make it work until seeing an article of mine on "the uniqueness of the core." (To this day, I don't see the connection.) Roddy's paper is one of the greatest in the theory of ordered sets [16].

Later, Roddy, Schröder, and Rutkowski proved that the answer is "Yes" even if just one of $P$ and $Q$ is finite, a farreaching extension, but the general problem is still open. Maybe you will solve it?

Duffus explored fixed points of automorphisms of direct products of posets when the posets were directly indecomposable. (An automorphism is an order-isomorphism from a poset to itself. A poset $P$ with $|P| \neq 1$ is directly indecomposable if, whenever $P \cong A \times B$ for posets $A$ and $B$, then $|A|=1$ or $|B|=1$.)

An interesting problem is to generalize Duffus's results. In particular,

Question 2. Let $P$ and $Q$ be connected posets such that every automorphism of $P$ or $Q$ has a fixed point. Does every automorphism of $P \times Q$ have a fixed point?
(See $\$ 2$ for the definition of "connected." We focus on connected posets because of a beautiful theorem that we will see in $\$ 2$.)

Bonus question. Does a truncated non-complemented lattice of finite height have the fixed-point property?

I have a dream... that one day I will be able to give a talk about lattices to a general audience without having to define "lattice." A lattice is a non-empty poset such that every pair of elements $x, y$ has a least upper bound, denoted $x \vee y$, and a greatest lower bound, $x \wedge y$. A poset with a least element 0 and a greatest element 1 is called bounded. A bounded lattice is complemented if for every element $x$, there is an element $y$ such that $x \vee y=1$ and $x \wedge y=0$. (These lattices have high self-esteem.) Examples include the power set of a set ordered by inclusion and the lattice of subspaces of a finite-dimensional vector space.

The complemented lattice $\mathbf{2} \times \mathbf{2}$ has the fixed-point property. If $f: \mathbf{2} \times \mathbf{2} \rightarrow \mathbf{2} \times \mathbf{2}$ is order-preserving, either $f(0,0)=(0,0)$ or $f(1,1)=(1,1)$ or $f(0,0)=f(1,1)$ equals (say) $(1,0)$ and hence $f(1,0)=(1,0)$. But if you remove the least and greatest elements, you get a poset without the fixed-point property, because the function switching $(1,0)$ and $(0,1)$ is order-preserving.

This doesn't happen with finite non-complemented lattices. Baclawski and Björner proved the following theorem.

Theorem 3. Let L be a finite, non-complemented lattice. Then the truncated lattice $L \backslash\{0,1\}$ has the fixed-point property.

Björner, a Pólya Prize winner, conjectured in 1981 that the theorem remains true even if $L$ is infinite, as long as there is a finite bound on the size of $L$ 's totally ordered subsets, called chains. (This is what it means for $L$ to have finite height.) This conjecture has been open since 1981. See [10].

\section{The Arithmetic of Ordered Sets}

Let $E$ and $X$ be posets. The set $E^{X}$ can be partially ordered, where, for $f, g \in E^{X}, f \leq_{E^{X}} g$ if $f(x) \leq_{E} g(x)$ for all $x \in X$.
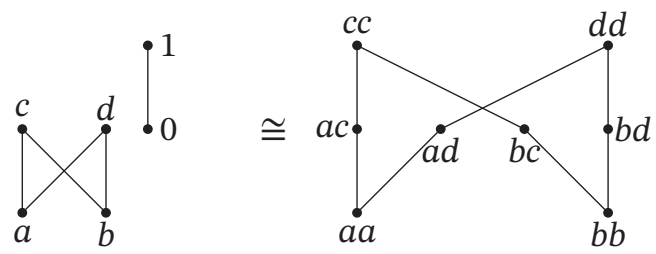

Figure 1. $E^{X}$, where $E$ is the 4-element cycle $\{a, b, c, d\}$ with $a, b<c, d$ and $X$ is the 2-element chain $\{0,1\} ; b c$ denotes the function $f: X \rightarrow E$ such that $f(0)=b$ and $f(1)=c$.

If $E$ and $X$ are disjoint, define the disjoint sum $E \amalg X$ of $E$ and $X$ to be the poset defined on $E \cup X$ such that $a \leq b$ in $E \coprod X$ if and only if $a, b \in E$ and $a \leq_{E} b$ or $a, b \in X$ and 
$a \leq_{X} b$. A non-empty poset that is not the disjoint sum of non-empty posets is connected.

In the 1979 Proceedings of the American Mathematical Society, Duffus and Wille proved that $P^{P} \cong Q^{Q}$ implies $P \cong Q$ if $P$ and $Q$ are both finite, non-empty, and connected [5, Theorem, p. 14].

Duffus-editor-in-chief of the journal Order from 2007 to 2015 and chair of the Mathematics Department at Emory University from 1991 to 2005-said in 1984, "It is still an open problem to show connectedness can be dropped." He notes in his 1978 thesis, "It is not obvious that $P$ is connected and $P^{P} \cong Q^{Q}$ imply that $Q$ is connected."

I proved that, indeed, if $P$ and $Q$ are finite, non-empty posets such that $P^{P} \cong Q^{Q}$ and $P$ is connected, then $Q$ is connected [8].

In other words, I resolved the issue from Duffus's 1978 thesis in that I have "half-dropped" the connectedness hypothesis used in the 1979 Proceedings of the American Mathematical Society paper, whereas the 1984 problem asked if it could be dropped entirely. That problem is still open for you to solve.

One of the most beautiful results in the theory of ordered sets is Hashimoto's Refinement Theorem: if $P$ is a connected, non-empty poset (finite or infinite!), any two ways of writing $P$ as a direct product (of a finite or infinite family of posets) have a common refinement. For example, if $P \cong A \times B \cong C \times D$, then there exist posets $W, X, Y, Z$ such that $A \cong W \times X, B \cong Y \times Z, C \cong W \times Y$, and $D \cong X \times Z$.

We can consider similar issues with respect to the exponentiation operator. If $E, X, Y$, and $Z$ are posets, then

$$
\left(E^{X}\right)^{Y \times Z} \cong E^{X \times Y \times Z} \cong\left(E^{Y}\right)^{X \times Z} .
$$

What if we have posets $A, B, C$, and $D$ such that $A^{C} \cong B^{D}$ ? Can we find "refining" posets $E, X, Y$, and $Z$ such that the isomorphism is naturally explained (by (দ)), where

$$
A \cong E^{X}, B \cong E^{Y}, C \cong Y \times Z \text {, and } D \cong X \times Z \text { ? }
$$

In 1982, Jónsson (an invited speaker at the 1974 International Congress of Mathematicians) and McKenzie, a professor at the University of California at Berkeley, posed the following problem. We only quote part of it [12].

"PROBLEM 12.1. Find counter examples (or prove that none exist) to the refinement of $A^{C} \cong B^{D}$ under any of the following conditions:...

(ii) $C, D$, and $A^{C}$ are finite and connected.

(iii) $A^{C}$ is a directly indecomposable lattice."

I solved Problem 12.1(ii) [9], but (iii) is still open. (I solved a problem of Jónsson and McKenzie related to (iii) in [6]; in that problem, Jónsson and McKenzie allowed one to assume the exponent was finite.)

Hashimoto's Refinement Theorem enables you to prove results like: if $P, Q$, and $R$ are finite, non-empty posets and $P \times R \cong Q \times R$, then $P \cong Q$. What if we merely have an order-embedding? This is a conjecture Birkhoff lists as a suggestion in a 1942 article.

Question 4. If $P, Q$, and $R$ are finite, non-empty posets and $P \times R$ is isomorphic to a subposet of $Q \times R$, is $P$ isomorphic to a subposet of $Q$ ?

It is unclear if Lovász's clever ideas in [13] would be applicable here.

\section{Free Distributive Lattices}

A lattice $L$ is distributive if for all $x, y, z \in L, x \wedge(y \vee z)=$ $(x \wedge y) \vee(x \wedge z)$. Distributive lattices are beautiful and wonderful. We will say that $(L, \iota: P \rightarrow L)$ is a free bounded distributive lattice generated by the finite poset $P$-we will denote the bounded distributive lattice $L$ by $\operatorname{FD}(P)$ if $\iota$ is order-preserving and, for any order-preserving map $\mu: P \rightarrow M$ from $P$ to a bounded distributive lattice $M$, there is a unique lattice-homomorphism $h: L \rightarrow M$ preserving 0 and 1 such that $h \circ \iota=\mu$. It turns out that $\operatorname{FD}(P)$ is order-isomorphic to $\mathbf{2}^{\mathbf{2}^{P}}[19$, p. 369].

Several famous problems in combinatorics can be rephrased in terms of free distributive lattices. For instance, the lattice $L(m, n)$ appears a lot in combinatorics: it is the poset $\left\{\left(a_{1}, \ldots, a_{m}\right) \in \mathbb{Z}^{m} \mid 0 \leq a_{1} \leq \cdots \leq a_{m} \leq n\right\}$ and it is order-isomorphic to $\operatorname{FD}(\mathbf{m}-\mathbf{1} \coprod \mathbf{n}-\mathbf{1})$. A still-open problem is to find a combinatorial proof that the polynomial $(1+x)\left(1+x^{2}\right) \cdots\left(1+x^{n}\right)$ is unimodal (this means that the coefficients form a unimodal sequence: they weakly increase, then weakly decrease); Stanley explained to me that this is tantamount to showing that a certain distributive lattice is unimodal (where the lattice is unimodal if the number of elements of each "height" yields a unimodal sequence of numbers). If one can prove combinatorially that $\mathrm{FD}(\mathbf{2} \times \mathbf{n})$ is unimodal, one will have solved this open problem.

If you look at a paper on "lozenge tilings of hexagons," you may see MacMahon's famous formula for counting "plane partitions in a box." This is the cardinality of $\mathrm{FD}(\mathbf{k} \coprod \mathbf{m} \coprod \mathbf{n})$ :

$$
\prod_{a=0}^{k} \prod_{b=0}^{m} \prod_{c=0}^{n} \frac{a+b+c+2}{a+b+c+1}
$$

I recall emailing Stanley circa 1992 to ask if there was a formula for the cardinality of $\mathrm{FD}(\mathbf{k} \coprod \mathbf{m} \coprod \mathbf{n} \coprod \mathbf{r})$ $(k, m, n, r \in \mathbb{N})$, and my memory is that he replied that it was a well-known open problem. It is still open [19, Chapter 3, Exercise 170(h)]. Stanley wrote in 1972, "Nothing significant seems to be known in general about" | $\mathrm{FD}(\mathbf{k} \coprod \mathbf{m} \coprod \mathbf{n} \coprod \mathbf{r}) \mid$.

Dedekind himself found that $\mathrm{FD}(\mathbf{1} \coprod \mathbf{1} \coprod \mathbf{1} \coprod \mathbf{1})$ has 168 elements, and the oldest problem in lattice theory is Dedekind's: find the cardinality of $\operatorname{FD}(\mathbf{1} \amalg \mathbf{1} \amalg \cdots \amalg \mathbf{1})$ where there are $n$ " 1 's," also known as the free distributive 
lattice with $n$ generators. This cardinality is known only for $n \leq 8$. Yamamoto proved that this cardinality is even if $n$ is even. What if $n$ is odd?

\section{Inequalities for h-vectors}

Let $\sigma=\sigma_{1} \sigma_{2} \ldots \sigma_{n}$ belong to the symmetric group $S_{n}$. A descent (an ascent) is an index $i \in\{1, \ldots, n-1\}$ such that $\sigma_{i}>\sigma_{i+1}$ (resp., $\sigma_{i}<\sigma_{i+1}$ ). A natural labeling of an $n$ element poset $P$ (also known as a natural labelling [7]) is an order-preserving bijection from $P$ to the chain $\{1,2, \ldots, n\}$. Figure 2(a) shows a natural labeling of the fence $F_{4}-F_{k}$ is the $k$-element poset $\left\{x_{1}, x_{2}, \ldots, x_{k}\right\}$ in which $x_{1}<x_{2}>x_{3}<$ $x_{4}>\cdots$-but Figure 2(b) does not.
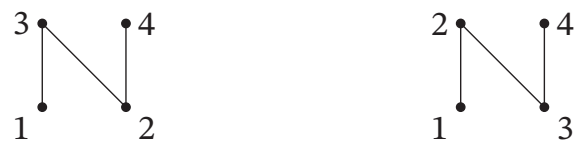

Figure 2. (a) A natural labeling. (b) An unnatural labeling.

If we fix a natural labeling, the linear extensions of $P$ listings of the elements of $P$ where $p$ appears to the left of $q$ if $p<q$ in $P$-are permutations. Every listing of the members of an $n$-element set is a permutation if one lists all the elements without repetition. Let $\mathrm{H}_{k}(P)$ be the set of linear extensions of $P$ with exactly $k$ descents, and let $\mathrm{h}_{k}(P)=\left|\mathrm{H}_{k}(P)\right|(0 \leq k \leq n)$. When the poset $P$ is understood, we will simply write " $\mathrm{h}_{k}$ " and " $\mathrm{H}_{k}$." One can have tremendous fun calculating $\mathrm{H}_{k}$. See Figure 3, where we partially order the linear extensions by saying that $\sigma_{1} \cdots \sigma_{i} \sigma_{i+1} \cdots \sigma_{n}<\sigma_{1} \cdots \sigma_{i-1} \sigma_{i+1} \sigma_{i} \sigma_{i+2} \cdots \sigma_{n}$ for $i$ an ascent and using transitivity. Note that $\mathrm{h}_{n} \equiv 0$.

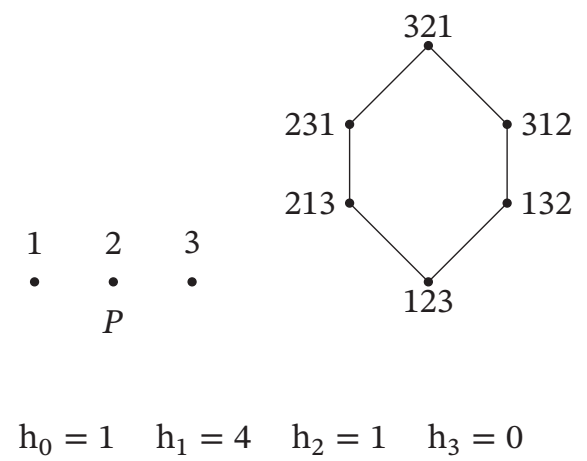

Figure 3(a).

What makes working with h-vectors-the $(n+1)$-tuples $\left(\mathrm{h}_{0}, \mathrm{~h}_{1}, \ldots, \mathrm{h}_{n}\right)$-so delightful is when there are two families of posets differing in structure that mysteriously have the same h-vectors. See Figures 4, 5, and 6 .

It is known that the h-vector of $\mathbf{2} \times \mathbf{n}$ is the sequence of Narayana numbers (see [19, Example 3.5.5]). In Table 1 , we show the h-vectors for the smallest posets of the

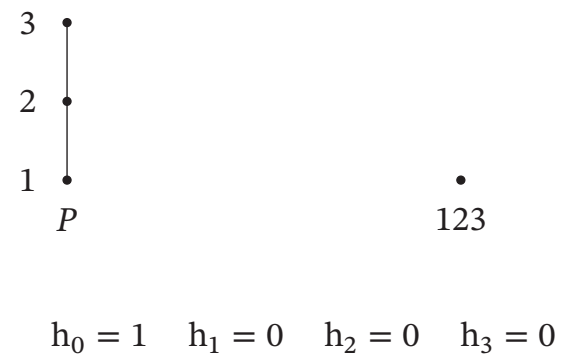

Figure 3(b).

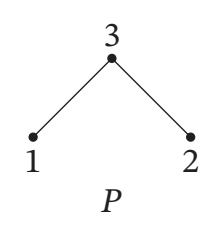

213

$$
\mathrm{h}_{0}=1 \quad \mathrm{~h}_{1}=1 \quad \mathrm{~h}_{2}=0 \quad \mathrm{~h}_{3}=0
$$

Figure 3(c).

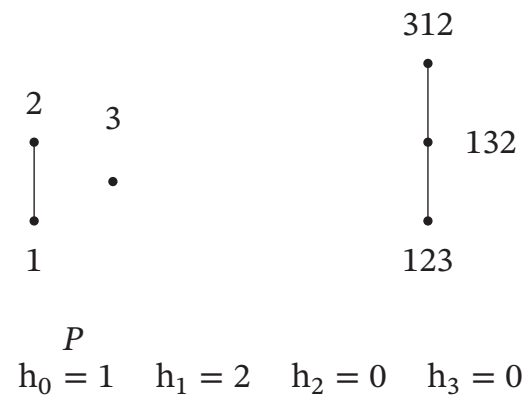

Figure 3(d).

$$
\begin{array}{ll}
\mathbf{2} \times \mathbf{1} & \overrightarrow{\mathrm{h}}=(1) \\
\mathbf{2} \times \mathbf{2} & \overrightarrow{\mathrm{h}}=(1,1) \\
\mathbf{2} \times \mathbf{3} & \overrightarrow{\mathrm{h}}=(1,3,1) \\
\mathbf{2} \times \mathbf{4} & \overrightarrow{\mathrm{h}}=(1,6,6,1) \\
\mathbf{3} \times \mathbf{1} & \overrightarrow{\mathrm{h}}=(1) \\
\mathbf{3} \times \mathbf{2} & \overrightarrow{\mathrm{h}}=(1,3,1) \\
\mathbf{3} \times \mathbf{3} & \overrightarrow{\mathrm{h}}=(1,10,20,10,1) \\
\mathbf{3} \times \mathbf{4} & \overrightarrow{\mathrm{h}}=(1,22,113,190,113,22,1) \\
\mathbf{3} \times \mathbf{5} & \overrightarrow{\mathrm{h}}=(1,40,400,1456,2212,1456,400,40,1)
\end{array}
$$

Table 1.

form $\mathbf{2} \times \mathbf{n}$ and $\mathbf{3} \times \mathbf{n}$. What properties do the "generalized Narayana" numbers $\mathrm{h}_{k}(\mathbf{3} \times \mathbf{n})$ have? (See the work of Robert Sulanke.) 


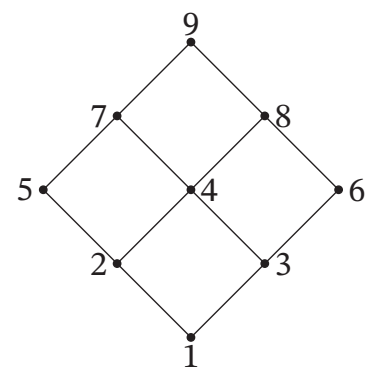

$\mathrm{h}_{0}=1 \quad \mathrm{~h}_{1}=10 \quad \mathrm{~h}_{2}=20 \quad \mathrm{~h}_{3}=10 \quad \mathrm{~h}_{4}=1$

Figure 4(a).

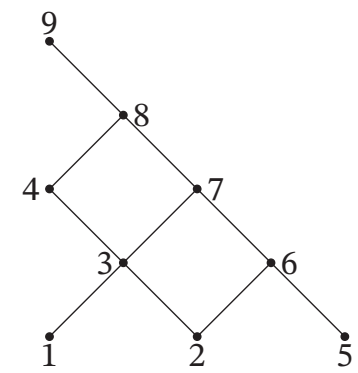

$\mathrm{h}_{0}=1 \quad \mathrm{~h}_{1}=10 \quad \mathrm{~h}_{2}=20 \quad \mathrm{~h}_{3}=10 \quad \mathrm{~h}_{4}=1$

Figure 4(b).

An answer should help resolve what Proctor calls "a complete mystery" [15, p. 555].

Readers will immediately formulate their own conjecture about h-vectors of two families of posets from Figures 5 and 6; MacMahon's formula, shown earlier, would yield it, but a bijective proof is lacking.

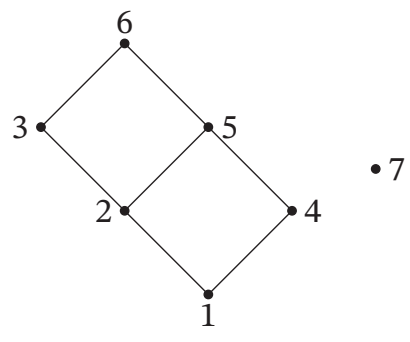

$$
\mathrm{h}_{0}=1 \quad \mathrm{~h}_{1}=12 \quad \mathrm{~h}_{2}=18 \quad \mathrm{~h}_{3}=4
$$

Figure 5(a).

$$
h_{0}=1 \quad h_{1}=12 \quad h_{2}=18 \quad h_{3}=4
$$

Figure 5(b).

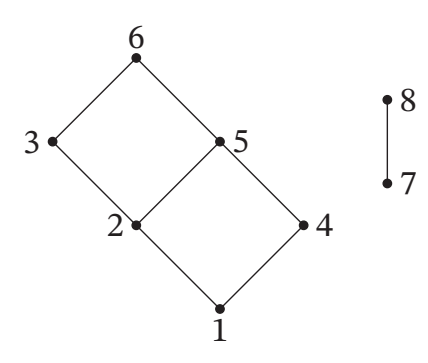

$$
\mathrm{h}_{0}=1 \quad \mathrm{~h}_{1}=21 \quad \mathrm{~h}_{2}=66 \quad \mathrm{~h}_{3}=46 \quad \mathrm{~h}_{4}=6
$$

Figure 6(a).

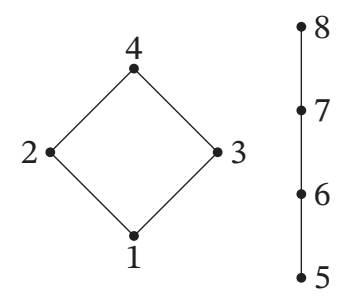

$$
\mathrm{h}_{0}=1 \quad \mathrm{~h}_{1}=21 \quad \mathrm{~h}_{2}=66 \quad \mathrm{~h}_{3}=46 \quad \mathrm{~h}_{4}=6
$$

Figure 6(b).

Can we combinatorially (i.e., with an explicit bijection or algorithm-in particular, without using the MacMahon formula) prove, for $\ell=0,1, \ldots, m n+m+n$, that

$\mathrm{h}_{\ell}(((\mathbf{m}+\mathbf{1}) \times \mathbf{n}) \coprod \mathbf{m})=\mathrm{h}_{\ell}(((\mathbf{n}+\mathbf{1}) \times \mathbf{m}) \coprod \mathbf{n})(\$ 137) ?^{1}$

(Professor Vaughan Pratt suggested this symmetric formulation in a discussion that started on the Universal Algebra Listserv.)

\footnotetext{
${ }^{1}$ The reader should be wondering if this is a typo or if the author is offering a cash prize for a solution to this problem.
} 
For $m=2$ and $n=3$, we are comparing

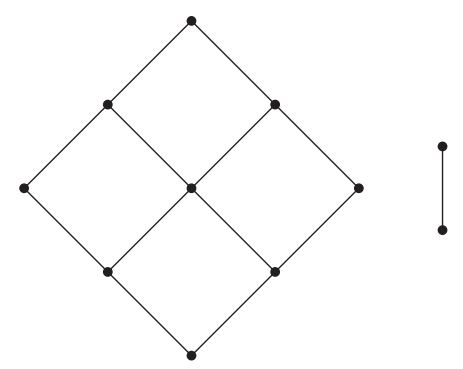

and

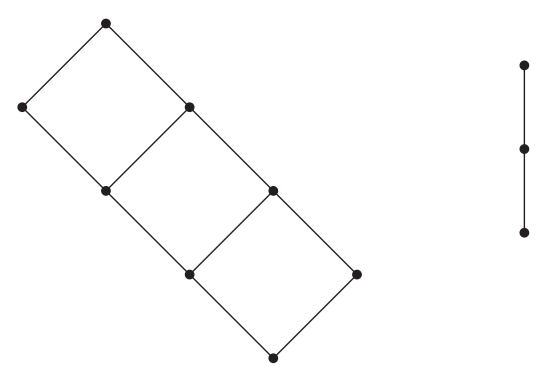

Going back to arbitrary $n$-element posets, I conjectured that, for $i \leq \frac{n-1}{2}, \mathrm{~h}_{n-1-i} \leq \mathrm{h}_{i}$. In 1997, Björner was able to prove the inequality using the $\mathrm{g}$-Theorem on polytopes, but, he wrote in a letter to me dated March 2, 1997, "This seems unnecessarily complicated. There should be a combinatorial proof! Namely, an injection from the set of linear extensions of $P$ with $[n-1-i]$ descents to [the set of linear extensions of $P$ with $i$ descents. It seems nontrivial however. Can you do it?"

I did it (unpublished), but there are other interesting inequalities between the entries of the $h$-vector that remain to be proved.

At the 1981 Banff Conference on Ordered Sets, Stanley said, "About ten years ago I proved" the following result.

Theorem 5 (Stanley). Let $P$ be a finite naturally labeled poset. Let $\mathcal{L}(P)$ be the set of linear extensions of $P$, and, for every $\pi \in \mathcal{L}(P)$, let $\mathrm{d}(\pi)$ be the number of descents of $\pi$. Let $M$ be $\max \{\mathrm{d}(\pi) \mid \pi \in \mathcal{L}(P)\}$. Then the following are equivalent:

(i) $\mathrm{h}_{i}=\mathrm{h}_{M-i}$ for $0 \leq i \leq M$.

(ii) Every maximal chain of $P$ has the same length.

He went on to pose the following problem:

"Find a combinatorial proof of this theorem. More precisely, when (ii) holds describe explicitly a bijection $f: \mathcal{L}(P) \stackrel{\simeq}{\rightarrow} \mathcal{L}(P)$ such that $\mathrm{d}(\pi)=M-\mathrm{d}(f(\pi))$ for all $\pi \in \mathcal{L}(P)$."

Stanley added, "It would even be interesting to do this for the case $P \cong " \mathbf{r} \times \mathbf{s}$.

Stanley reported in his 2012 book [19, p. 454], "A combinatorial proof using a complicated recursion argument was given by J. D. Farley" [7]. A more direct proof might help us prove that $\mathrm{h}_{k} \leq \mathrm{h}_{M-k}$ for $k \leq \frac{M}{2}$, part of a conjecture of Hibi, a winner of the Algebra Prize of the Mathematical Society of Japan.

\section{The Structure of the Perfect Terrorist Cell}

In 2008, policeman Kevin Blake, the former director of Jamaica's National Intelligence Bureau, part of Jamaica's Ministry of National Security, told the Jamaica Observer newspaper, "Ordered sets theory allows you to organise networks, a criminal network or a terrorist cell, and mathematically calculate the chances of successfully disrupting the cell upon capturing a number of those individuals." The newspaper went on to say, "Blake's work has already contributed to the disruption of one criminal gang."

Whatever you might think of this work, maybe it played a role in a former deputy director of the CIA's calling me up and having coffee with me. It's easy to get a big head when a US Air Force officer (now a colonel) says that your "thinking has influenced real world decisions of highest operational importance to the Department of Defense" or when the Wiener Zeitung does a two-page profile of you or when Seed Magazine names you one of "15 people who have shaped the global conversation about science in 2005" (alongside people like Harvard University physics professor Lisa Randall). Fortunately, I have remained modest.

Zeinab Bandpey (shown in Figure 7) found the structure of the "perfect" terrorist cell with a single leader, according to certain specific criteria. Mathematically, she

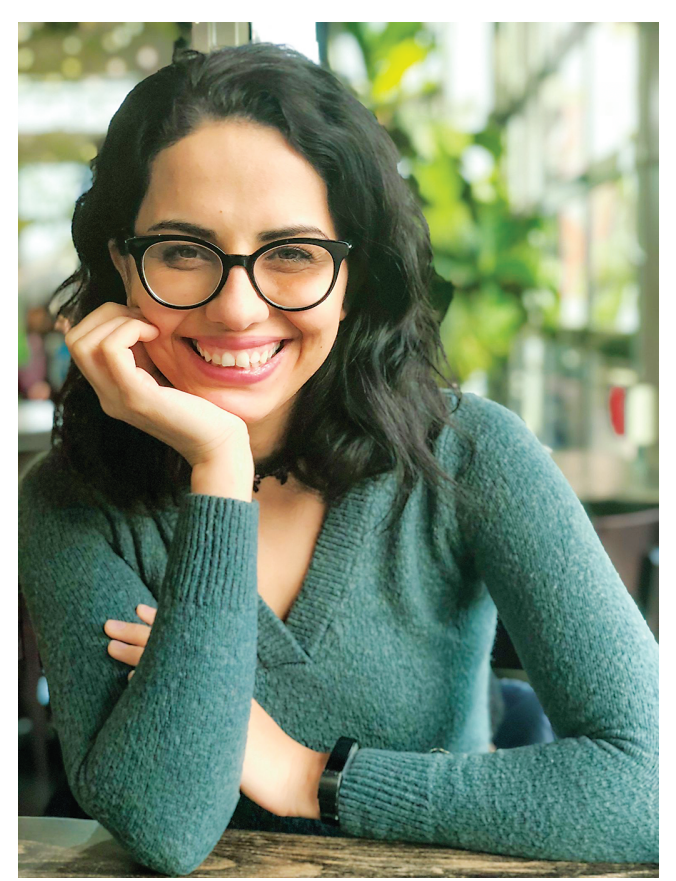

Figure 7. Zeinab Bandpey. 
proved a conjecture about minimizing the number of cutsets-subsets that intersect all maximal chains-in a finite poset $P$ with a top element such that no element has more than $b$ lower covers [1].

Before Bandpey's breakthrough, Chvátal and three colleagues at McGill University had previously proven a special case of the conjecture for trees (the chairman of Stanford University's Computer Science Department at one time called Chvátal "one of the two best young combinatorialists in the world") and I had proven the $b=2$ case.

What if $P$ has two maximal elements (or more)?

\section{6. "Trying to Reestablish the Cosmic Order by Riding on a Buffalo"}

While I don't necessarily agree with "Professor Gelfand's oft-repeated prediction that lattice theory will play a leading role in the mathematics of the twenty-first century" (as Rota said in the Notices [17, p. 1445]), lattice theory and the theory of ordered sets arise in a variety of applications, from robotics [4] to imaging to chemistry to linguistics to economics [20] (see Roth's Nobel Prize acceptance speech) to sociology to epidemiology [2] to physics [3] to national security (the work of Cliff Joslyn at Los Alamos National Laboratory predates mine) to computer science [14]. ${ }^{2}$ But I will leave you with a list of 12 problems in pure mathematics:

(0) If $P$ and $Q$ are posets with the fixed-point property, does $P \times Q$ have the fixed-point property? If every automorphism of the connected poset $P$ (resp., $Q$ ) has a fixed point, does every automorphism of $P \times$ $Q$ have a fixed point?

(1) Let $P$ and $Q$ be finite, non-empty posets. If $P^{P} \cong$ $Q^{Q}$, is $P \cong Q$ ?

(2) Let $P, Q$, and $R$ be finite, non-empty posets. If $P \times R$ embeds in $Q \times R$, does $P$ embed in $Q$ ?

(3) Let $L$ be an exponentially and directly indecomposable lattice, and let $P$ be a connected, nonempty poset. Is the automorphism group of $L^{P}$ isomorphic to the product of the automorphism group of $L$ and the automorphism group of $P$ ? (A poset $P$ is exponentially indecomposable if, whenever $P \cong A^{B}$ for posets $A$ and $B$, then $|B|=1$.)

(4) Find a formula for the cardinality of the free distributive lattice generated by a disjoint sum of four finite chains.

(5) If $n$ is odd, when is the cardinality of the free distributive lattice with $n$ generators odd?

\footnotetext{
${ }^{2}$ See the list of talks in the AMS Special Sessions on The Many Lives of Lattice Theory and the Theory of Ordered Sets for 2002, 2003, and 2004, as well as the DIMACS Workshop on Applications of Lattices and Ordered Sets to Computer Science and the DIMACS Workshop on Applications of Order Theory to Homeland Defense and Computer Security.
}

(6) Without using the MacMahon formula (and preferably with a bijection), prove that, for $\ell \in \mathbb{N}$, $\mathrm{h}_{\ell}(((\mathbf{m}+\mathbf{1}) \times \mathbf{n}) \amalg \mathbf{m})=\mathrm{h}_{\ell}(((\mathbf{n}+\mathbf{1}) \times \mathbf{m}) \amalg \mathbf{n})$ (\$137). (Remember, this is a prelude to explaining combinatorially why the h-vectors of posets in the classes containing the posets of Figures 4(a) and (b) are the same.)

(7) Generalize all the results we know about Narayana numbers to $\mathrm{h}_{k}(\mathbf{m} \times \mathbf{n})$.

(8) Let $P$ be a finite poset and let $M$ be the largest integer such that $\mathrm{h}_{M}(P) \neq 0$. Is $\mathrm{h}_{k} \leq \mathrm{h}_{M-k}$ if $k \leq \frac{M}{2}$ ?

(9) In the class of finite posets with $m$ maximal elements such that no element has more than $b$ lower covers, find the poset or posets with the fewest cutsets.

And to show that there is more than we could discuss in this article:

(10) Are $Y$ and $Z(r)$ the only directly indecomposable differential lattices? [18, Problem 2]

(11) Is every finite lattice the lattice of congruences of a finite algebra? [11]

I hope you solve them.

Fin

ACKNOWLEDGMENTS. The author thanks Professor Asamoah Nkwanta, Professor Bernd Schröder, the referees, and the editor for critiquing a draft of this article. Notices policy forced me to restrict the number of references to 20. The author is thankful for the opportunity to contribute to the Notices but objects to one editorial decision.

\section{References}

[1] Zeinab Bandpey, Jonathan Farley's mathematical terror theory: the structure of perfect terrorist cells with a single leader, Congr. Numer. 228 (2017), 345-352. MR3751850

[2] Niko Beerenwinkel, Nicholas Eriksson, and Bernd Sturmfels, Evolution on distributive lattices, J. Theoret. Biol. 242 (2006), no. 2, 409-420, DOI 10.1016/j.jtbi.2006.03.013 MR2272562

[3] Garrett Birkhoff and John von Neumann, The logic of quantum mechanics, Ann. of Math. (2) 37 (1936), no. 4, 823843, DOI 10.2307/1968621 MR1503312

[4] Domitilla Del Vecchio and Richard M. Murray, Existence of cascade discrete-continuous state estimators for systems on a partial order, Hybrid Systems: Computation and Control, Lecture Notes in Computer Science, vol. 3414, Springer, Berlin, 2005, pp. 226-241.

[5] Dwight Duffus and Rudolf Wille, A theorem on partially ordered sets of order-preserving mappings, Proc. Amer. Math. Soc. 76 (1979), no. 1, 14-16, DOI 10.2307/2042907 MR534380 
[6] J. D. Farley, The automorphism group of a function lattice: a problem of Jónsson and McKenzie, Algebra Universalis 36 (1996), no. 1, 8-45, DOI 10.1007/BF01192707. MR1397566

[7] Jonathan David Farley, Linear extensions of ranked posets, enumerated by descents. A problem of Stanley from the 1981 Banff conference on ordered sets, Adv. in Appl. Math. 34 (2005), no. 2, 295-312, DOI 10.1016/j.aam.2004.05.007. MR2110553

[8] Jonathan David Farley, An issue raised in 1978 by a thenfuture editor-in-chief of the journal Order: Does the endomorphism poset of a finite connected poset tell us that the poset is connected?, arXiv:2005.03255, 2020.

[9] Jonathan David Farley, Another problem of Jónsson and McKenzie from 1982: refinement properties for connected powers of posets, Algebra Universalis 82 (2021), no. 3, Paper No. 48, 6, DOI 10.1007/s00012-020-00698-y. MR4289456

[10] Jonathan David Farley, A conjecture of Kozlov from the 1998 Proceedings of the American Mathematical Society: Non-evasive order complexes and generalizations of noncomplemented lattices, manuscript, 2020.

[11] Walter Feit, An interval in the subgroup lattice of a finite group which is isomorphic to $M_{7}$, Algebra Universalis 17 (1983), no. 2, 220-221, DOI 10.1007/BF01194532. MR726276

[12] Bjarni Jónsson and Ralph McKenzie, Powers of partially ordered sets: cancellation and refinement properties, Math. Scand. 51 (1982), no. 1, 87-120, DOI 10.7146/math.scand.a11966 MR681261

[13] L. Lovász, Operations with structures, Acta Math. Acad. Sci. Hungar. 18 (1967), 321-328, DOI 10.1007/BF02280291. MR214529

[14] G. Markowsky and B. K. Rosen, Bases for chain-complete posets, IBM J. Res. Develop. 20 (1976), no. 2, 138-147, DOI $10.1147 /$ rd.202.0138. MR392706

[15] Robert A. Proctor, Shifted plane partitions of trapezoidal shape, Proc. Amer. Math. Soc. 89 (1983), no. 3, 553-559, DOI 10.2307/2045516, MR715886

[16] Michael S. Roddy, Fixed points and products, Order 11 (1994), no. 1, 11-14, DOI 10.1007/BF01462225. MR1296230

[17] Gian-Carlo Rota, The many lives of lattice theory, Notices Amer. Math. Soc. 44 (1997), no. 11, 1440-1445. MR1488572

[18] Richard P. Stanley, Differential posets, J. Amer. Math. Soc. 1 (1988), no. 4, 919-961, DOI 10.2307/1990995. MR941434

[19] Richard P. Stanley, Enumerative combinatorics. Volume 1, 2nd ed., Cambridge Studies in Advanced Mathematics, vol. 49, Cambridge University Press, Cambridge, 2012. MR2868112

[20] Qingyun Wu and Alvin E. Roth, The lattice of envy-free matchings, Games Econom. Behav. 109 (2018), 201-211, DOI 10.1016/j.geb.2017.12.016 MR3802588

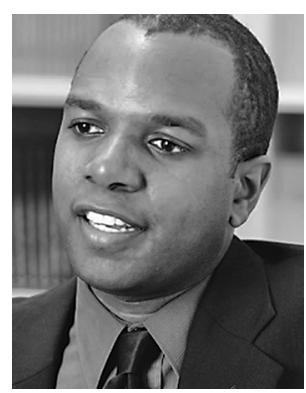

Jonathan David

Farley

Credits

Opening image is courtesy of Marlena Hewitt.

Figures 1-6 and Table 1 are courtesy of Jonathan David Farley. Figure 7 is courtesy of Elyad Farajnia.

Photo of Jonathan David Farley is courtesy of Peter Kiar.

\section{AMS AUTHOR} RESOURCE CENTER

The Author Resource Center is a collection of

information and tools available to assist you to

successfully write, edit, illustrate, and publish

your mathematical works.

To begin utilizing

these important

resources, visit:

www.ams.org/authors

O
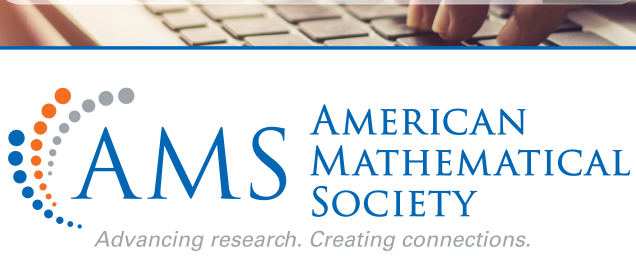